Supporting Information

\title{
Artificial Nacre Epoxy Nanomaterials based on Janus Graphene Oxide for Thermal Management Applications
}

Yang Wang, Zheng Zhang, Ting Li, Piming Ma, Xuhui Zhang, Bihua Xia, Mingqing Chen, Mingliang Du, Tianxi Liu, Weifu Dong*

Key Laboratory of Synthetic and Biological Colloids, Ministry of Education, School of Chemical and Material Engineering, Jiangnan University, 1800 Lihu Road, Wuxi 214122, China;

*E-mail: wfdong@jiangnan.edu.cn

Total pages: 4 (S1-S3)

\section{Experimental Section}

preparation of graphene oxide $(\mathrm{GO})$

\section{Figures}

Figure S1. (a) AFM image of GO layers on Si substrate. (b) Raman spectra taken at selected GO with the different numbers of layers (The numbers 1, 2, 3, 4, 5 are in correspondence with the numbers in AFM). (c) The intensity ratio $I(\mathrm{D}+\mathrm{G}) / I(2 \mathrm{TO}(\mathrm{Si}))$ as a function of the number of layers GO.

Figure S2. The cross-section images of (a1) GO and (b1) PGO scaffolds. (a2) E-GO and (b2) EPGO nanocomposites, respectively.

Figure S3. The cross-section ( $a$ and $b$ ) and side-view (c) morphology of JPGO scaffolds.

\section{Experimental details for preparation of graphene oxide (GO).}

In a typical procedure, graphite powder $(6 \mathrm{~g})$ was first treated with a mixture of $\mathrm{H}_{2} \mathrm{SO}_{4}(180$ $\mathrm{mL})$ and $\mathrm{HNO}_{3}(60 \mathrm{~mL})$ at room temperature for $24 \mathrm{~h}$ resulting in graphite intercalated compounds. Subsequently, expanded graphite (EG) was obtained by thermally expanding the dry graphite intercalated compounds powder at $1000^{\circ} \mathrm{C}$ for $10 \mathrm{~s}$. The EG $(2.0 \mathrm{~g})$ was pre-oxidized by vigorous 
stirring at $40^{\circ} \mathrm{C}$ in a mixture containing $\mathrm{H}_{2} \mathrm{SO}_{4}(360 \mathrm{~mL})$ and $\mathrm{H}_{3} \mathrm{PO}_{4}(40 \mathrm{~mL})$. Under vigorous stirring, $\mathrm{KMnO}_{4}(9.0 \mathrm{~g})$ was added slowly to the mixture. Then the mixture was heated to $50^{\circ} \mathrm{C}$ and stirred for $12 \mathrm{~h}$ to yield graphite oxide. This product was purified by seven cycles of wash with $\mathrm{HCl}$ solution $(3 \% 200 \mathrm{~mL})$ and deionized water. Then dry GO sheets was achieved under reduced pressure overnight at room temperature.
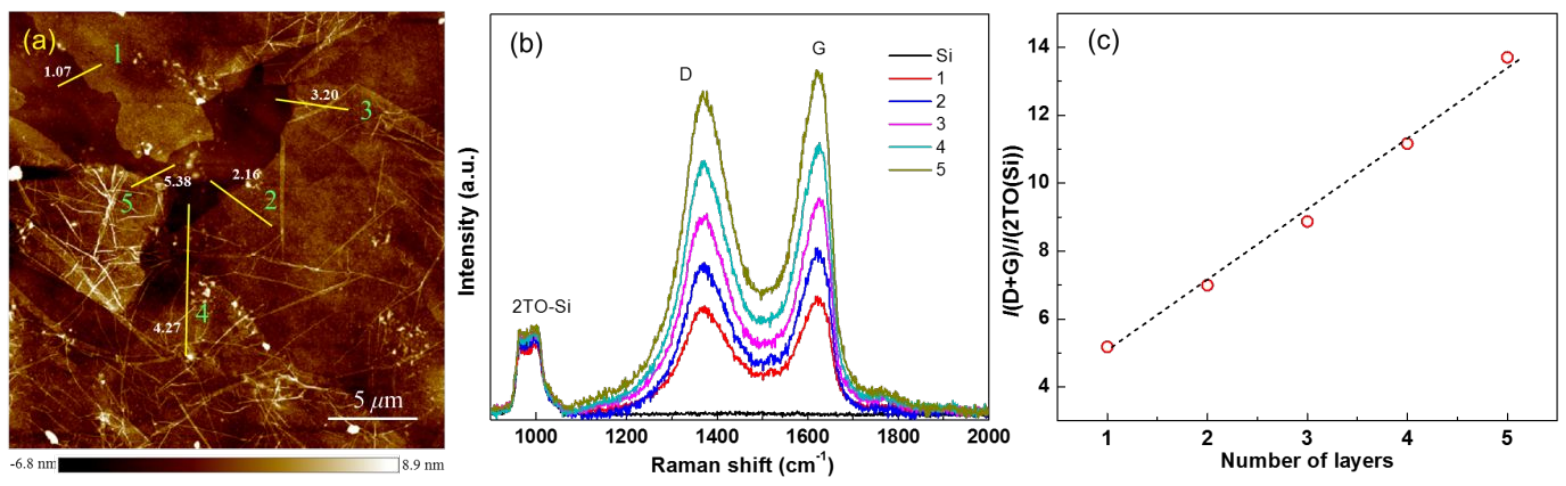

Figure S1. (a) AFM image of GO layers on Si substrate. (b) Raman spectra taken at selected GO with the different numbers of layers (The numbers $1,2,3,4,5$ are in correspondence with the numbers in $\mathrm{AFM})$. (c) The intensity ratio $I(\mathrm{D}+\mathrm{G}) / I(2 \mathrm{TO}(\mathrm{Si}))$ as a function of the number of layers GO.

Raman spectroscopy was used to identify the layers of the GO. The GO layers was deposited on Si substrates by spin coasting method. Figure S1 presents AFM image of GO sheets on a $\mathrm{Si}$ substrate. The GO sheets show five areas of different contrast, which are likely due to five different thicknesses. The thicknesses of the GO sheets are shown in Figure S1a. The thickness of singlelayer GO sheet is observed to be approximately $1.07 \mathrm{~nm}$. As the number of layers increases, the thickness linearly increases. For example, 2.16, 3.20, 4.27 and $5.38 \mathrm{~nm}$ correspond to two, three, four and five layers, respectively. Figure S1b displays the typical Raman spectra of a GO sheet 
with a single-layer to five-layer sample, which were measured under the same conditions. The two main peaks observed at $\sim 1370$ and $1619 \mathrm{~cm}^{-1}$ are the $\mathrm{D}$ and $\mathrm{G}$ band, respectively. ${ }^{[1]}$ As shown in Figure S1c, the intensity ratio $I(\mathrm{D}+\mathrm{G}) / I(2 \mathrm{TO}(\mathrm{Si}))$ increases linearly as the number of GO layers increases, which is similar to observations reported in previous work. ${ }^{[1,2] "}$

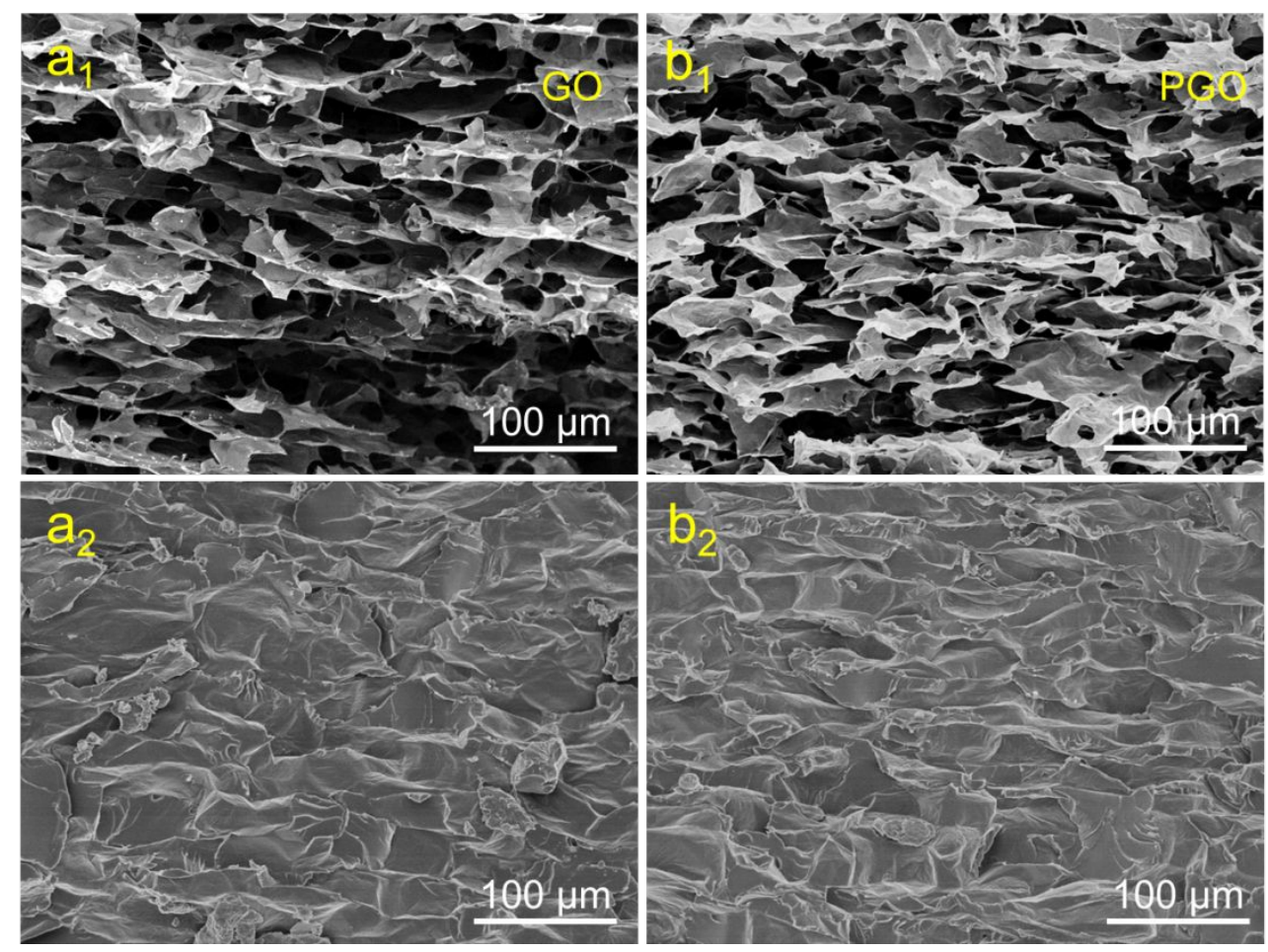

Figure S2. The cross-section images of (a1) GO and (b1) PGO scaffolds. (a2) E-GO and (b2) EPGO nanocomposites, respectively.

As shown in Figure S2, The PGO or GO scaffold shows a same three-dimensional interlocked and layered structure.
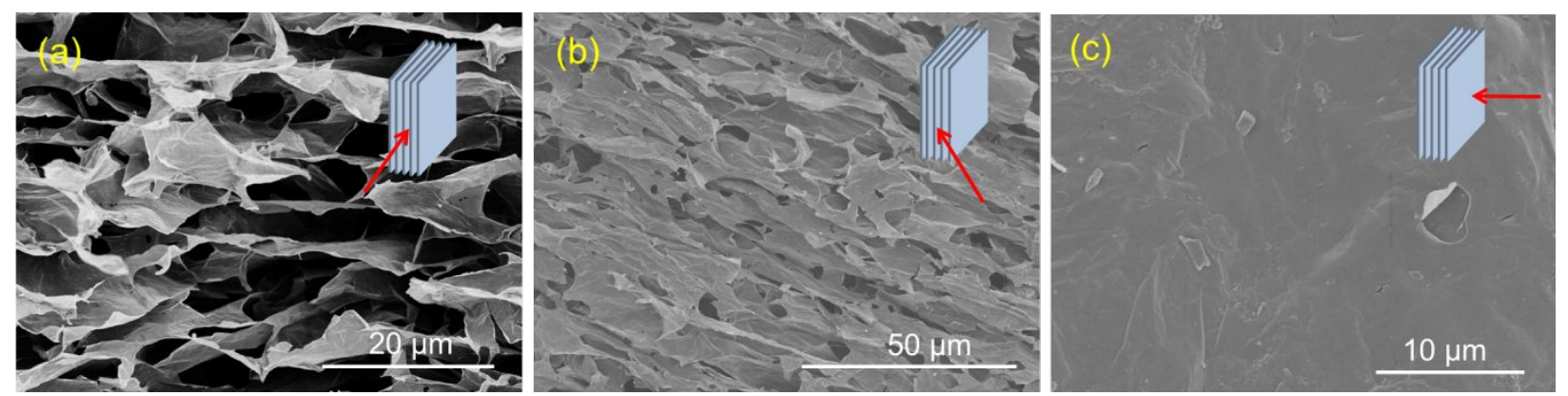
Figure S3. The cross-section (a and b) and side (c) morphology of JPGO scaffolds.

Different-view morphology of JPGO scaffolds were shown in Figure S3. The JPGO scaffold shows an oriented and porous structure at the cross-section ( $a$ and $b$ ) and a large lamella on the side of this scaffold (c). Consequently, this JPGO scaffold presents an anisotropic architecture.

\section{REFERENCES}

[1] Kostiuk, D.; Bodik, M.; Siffalovic, P.; Jergel, M.; Halahovets, Y.; Hodas, M.; Pelletta, M.; Pelach, M.; Hulman, M.; Spitalsky, Z. Reliable Determination of the Few-Layer Graphene Oxide Thickness Using Raman Spectroscopy. J. Raman Spectrosc. 2016, 47, 391-394.

[2] Tang, H.; Hu, H.; Wang Y.; Yu, T. Rapid and Non-Destructive Identification of Graphene Oxide Thickness Using White Light Contrast Spectroscopy. Carbon 2013, 53, 528-534. 